\title{
Time to antithrombotic therapy at Monash Health among patients with ischemic stroke and transient ischaemic attack (TIA)
}

Thanh G Phan, Chelsea Matley, Talvika Kooblal, Clissold Benjamin, Shaloo Singhal, John Ly, Mandy Thrift, Velandai
Srikanth, Henry Ma

Department of Medicine, Monash University, Stroke Unit, Monash Health, Department of Medicine, Peninsular Health

\section{Introduction}

There has been emphasis on timely administration of thrombolysis and clot retrieval but not antithrombotic therapy for ischemic stroke.

The frequency of antithrombotic therapy administration within 48 hours among patients with transient ischemic attack (TIA) was $73 \%$ in Australia, while among patients with ischemic stroke this ranged from $21 \%$ in New Zealand, 64\% in Australia to $95 \%$ in North America.

Aim: We plan to assess the time to antithrombotic therapy at Monash Medical Centre.

\section{Methods}

This is a retrospective study of admissions to the stroke unit at Monash Health over 6 months in 2015.

We collected data on demographic variables, admission diagnoses, time to triage, imaging, and time to administration of antithrombotics.

Statistical analyses were performed with survival package in $\mathrm{R}$ to plot the cumulative event of patients receiving antithrombotic therapy against the time to administration.

\section{Results}

There were 161 patients $(52.2 \%$ male, age $69.8 \pm 17.6$ years). Of these 101 patients had an ischaemic event, TIA $(n=19)$ or ischaemic stroke $(\mathrm{n}=87)$. Sixtyfive percent of the patients were kept nil by mouth on admission. The average time to imaging was $0.1 \pm 0.3$.days. The average time to dysphagia screen was

Figure 1

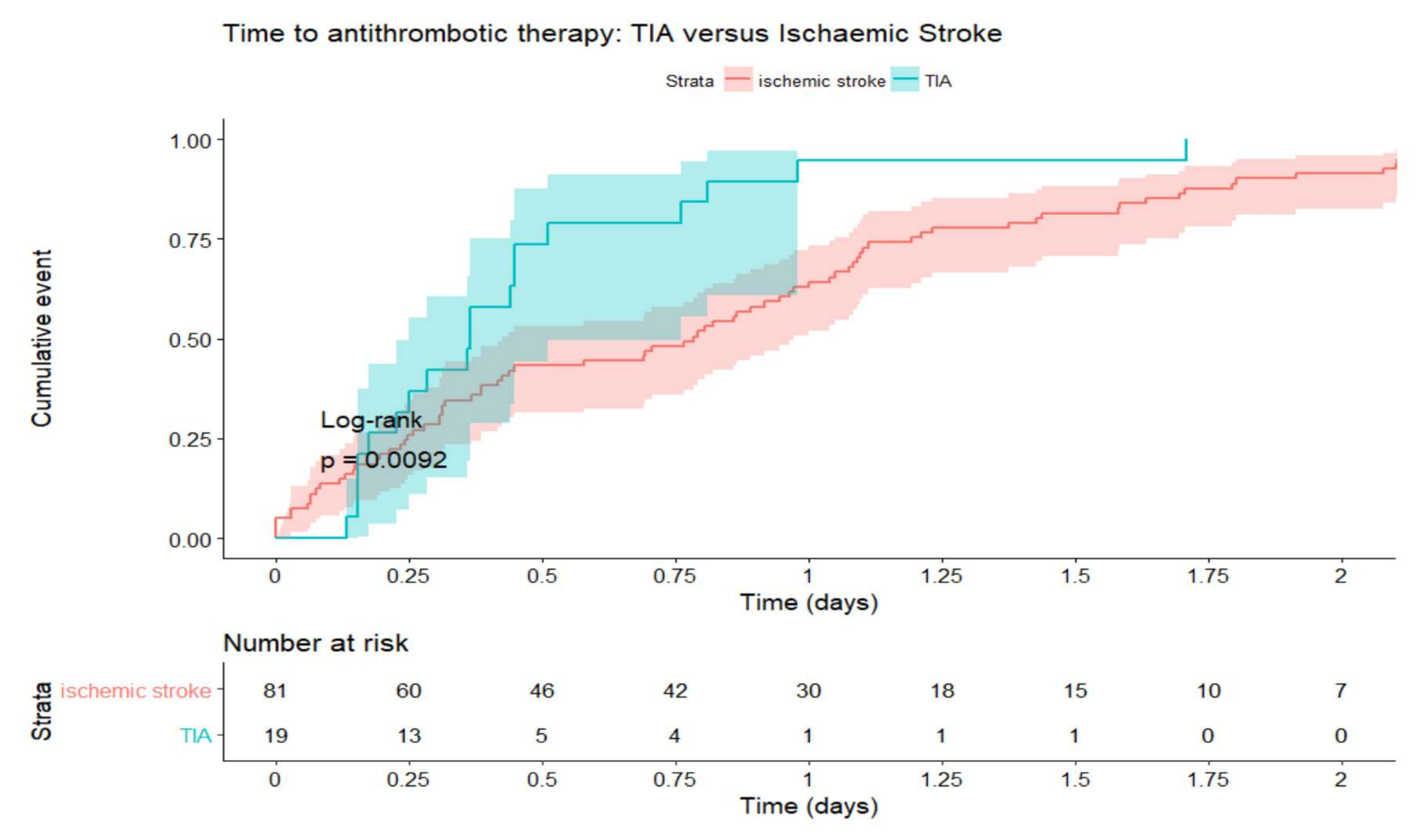

Figure 2

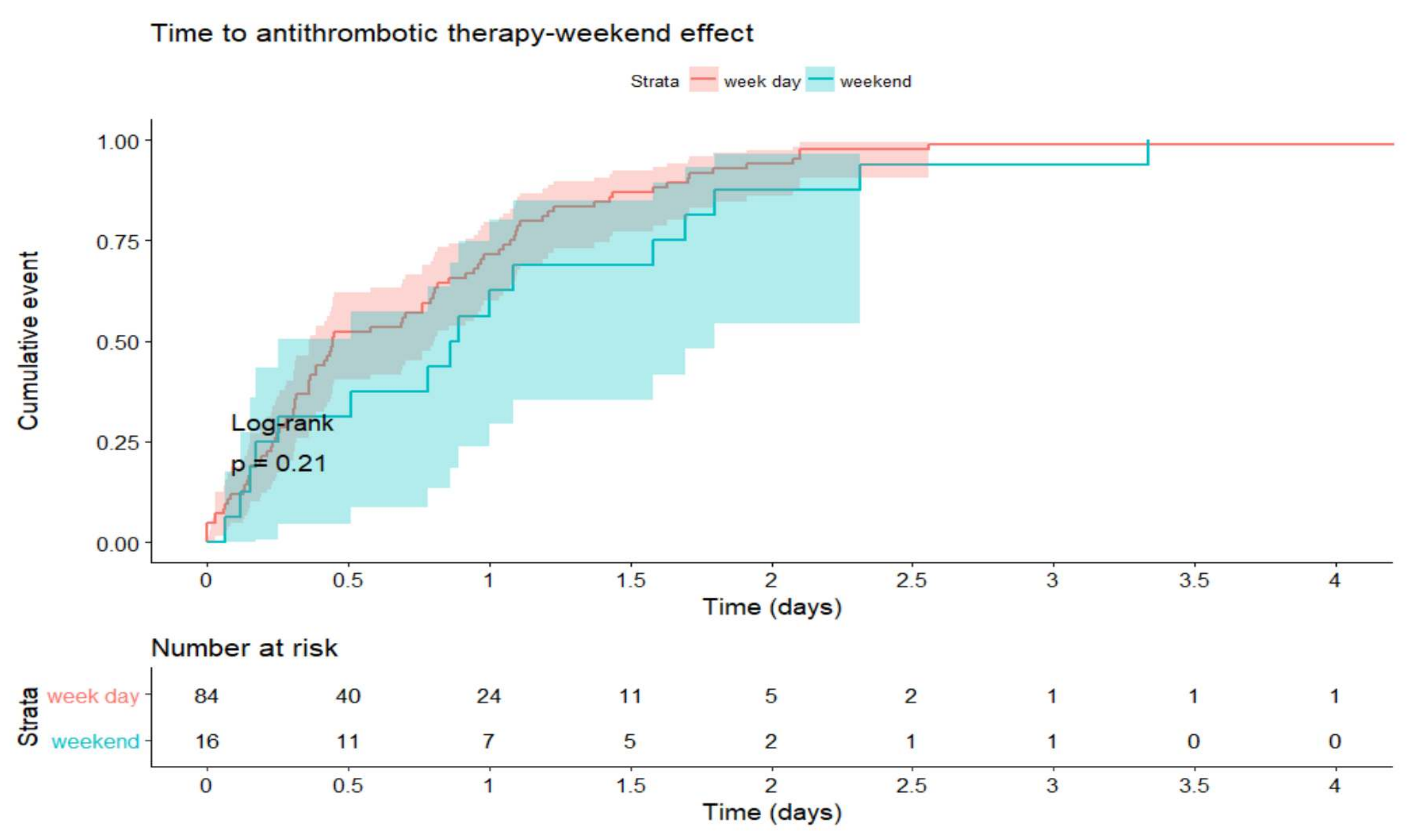

Figure 3

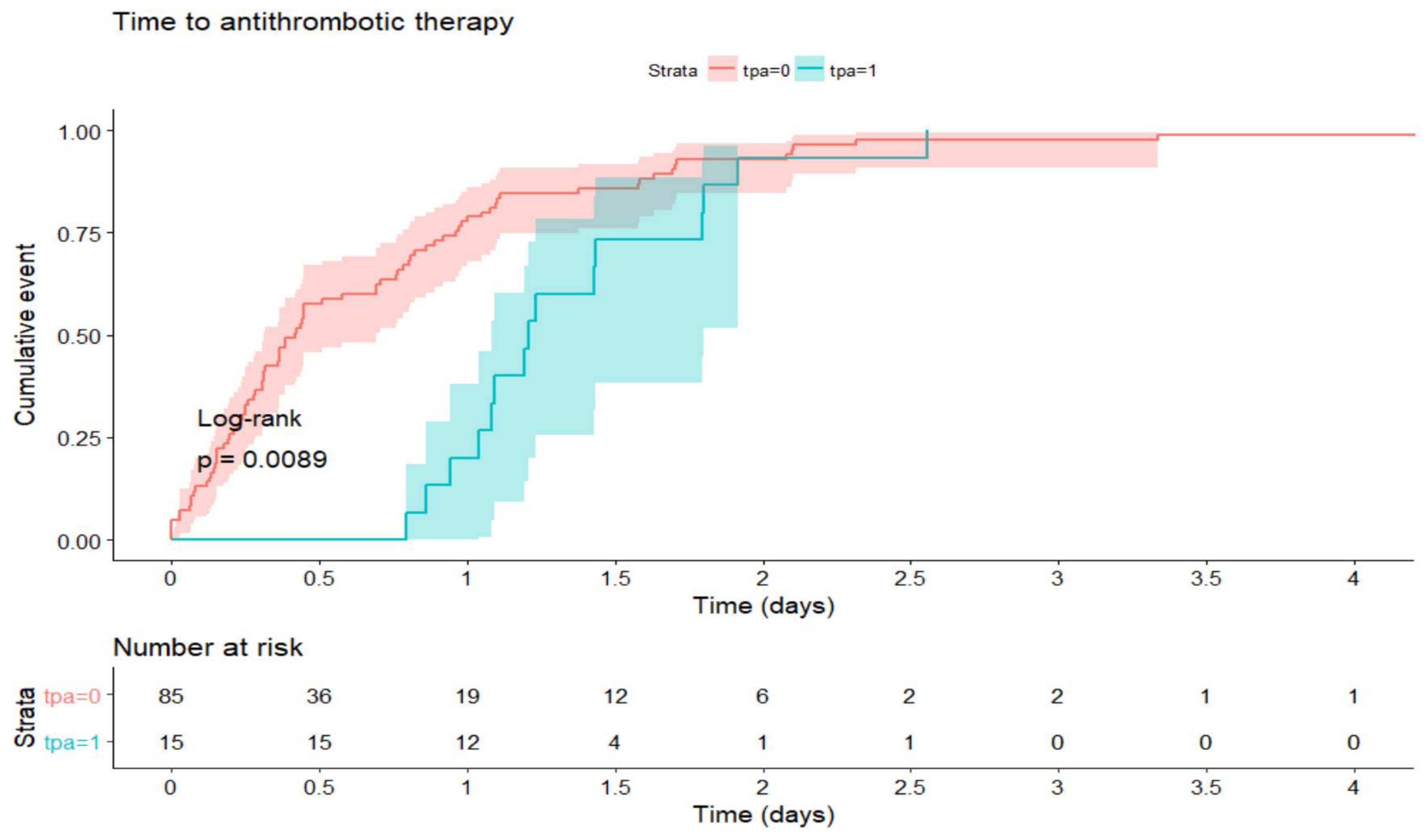

Results

Of those with ischaemic stroke, $65.5 \%$ of the patients were started on antithrombotic therapy by 24 hours and $91.9 \%$ by 48 hours. Of those with TIA, 73.7\% received antithrombotic therapy within 12 hours, $94.7 \%$ within 24 hours and $100 \%$ at 48 hour. TIA patients received antithrombotics earlier than those with ischemic stroke ( $\mathrm{p}=0.009$, see Figure 1) .

There was no statistical significant difference between week day and weekend (Figure 2) or office hour and after hour (not shown).

The use of thrombolytic therapy was associated with delay in commencing antithrombotic therapy ( $\mathrm{p}=0.009)$ (Figure 3). This is not unexpected since the protocol requires waiting for 24 hour after thrombolytic drugs.

\section{Limitations}

This is a retrospective study. The results should be considered as preliminary at this stage.

\section{Conclusion}

The proportion of patients with ischaemic stroke or TIA

receiving antithrombotic therapy within 48 hours was higher than previously reported in Australia and New Zealand

Our data are comparable to the high standard set by North American hospitals.

\section{Reference}

1-Schwamm et al. Get With the Guidelines-Stroke Is Associated With Sustained Improvement in Care for Patients Hospitalized With Acute Stroke or Transient Ischemic Attack. Circulation 2008 $0.8 \pm 1.2$ days. The average time to antithrombotic was $0.8 \pm 0.8$ 